\title{
INDUCED MATROIDS
}

\section{R. A. BRUALDI ${ }^{1}$}

\begin{abstract}
There are several known results concerning how matroids can be induced from given matroids by a bipartite graph and the properties that are inherited in this way. The purpose of this note is to extend some of these results to the situation where the bipartite graph is replaced by an arbitrary directed graph. We show how a directed graph and a matroid can be used to induce a new matroid. If the initial matroid is strongly base orderable, we prove that the induced matroid is also. In particular, a matroid induced from a free matroid by a directed graph is strongly base orderable. A consequence is that the cycle matroid of the complete graph on four nodes cannot be induced from a free matroid by any directed graph.
\end{abstract}

1. Let $\Gamma$ be a directed graph in which each edge joins two distinct nodes. A finite linear path $\theta$ is a finite sequence $\left(x_{1}, x_{2}, \cdots, x_{n}\right)$ of distinct nodes where $n \geqq 1$ and $\left(x_{i}, x_{i+1}\right)$ is an edge of $\Gamma(1 \leqq i<n)$. Notice that for each node $x,(x)$ is a finite linear path which we refer to as degenerate. The initial node of the path $\theta$ is $x_{1}$, denoted by $\operatorname{In} \theta$, while the terminal node of $\theta$ is $x_{n}$, denoted by Ter $\theta$; the set of nodes of $\theta$ is $\operatorname{Nod} \theta=\left\{x_{1}, x_{2}, \cdots, x_{n}\right\}$. If $\Theta$ is a collection of paths in $\Gamma$, then In $\Theta=\{$ In $\theta: \theta \in \Theta\}$, Ter $\Theta=\{$ Ter $\theta: \theta \in \Theta\}$, and Nod $\Theta=$ $\{\operatorname{Nod} \theta: \theta \in \Theta\}$. We say $\Theta$ is a set of pairwise node disjoint paths if $\theta_{1}$, $\theta_{2} \in \Theta, \theta_{1} \neq \theta_{2}$ imply Nod $\theta_{1} \cap \operatorname{Nod} \theta_{2}=\varnothing$.

We formulate here the rule used in [3] for obtaining a theorem about pairwise node disjoint paths in a directed graph from the corresponding theorem for bipartite graphs. (Alternately, one can use the second approach of [3] to obtain directly the same result.) This rule can be formulated for infinite sets of paths as in [3], but we shall restrict our attention to finite sets of paths, although the directed graph $\Gamma$ may be infinite. We shall then use this rule to prove some theorems about matroids.

2. Let $\Theta, \Phi$ be two finite sets of pairwise node disjoint finite linear paths in the directed graph $\Gamma$. Let each node of Nod $\Theta \cap$ Nod $\Phi$ be called an intersection node. The intersection nodes divide the paths of

Received by the editors October 19, 1970.

A MS 1970 subject classifications. Primary 05B35, 05C20; Secondary $05 \mathrm{~A} 05$.

Key words and phrases. Matroid, directed graph, induced matroid, free matroid, cycle matroid, bipartite graph; pairwise node disjoint paths, base orderable matroid, strongly base orderable matroid.

${ }^{1}$ Research supported by NSF grant GP-17815. 
$\Theta$, resp. $\Phi$, into a collection $\Sigma \Theta$, resp. $\Sigma \Phi$, of subpaths as follows. Let $\theta=\left(x_{1}, x_{2}, \cdots, x_{n}\right) \in \Theta$. If no node of $\theta$ is an intersection node, then $\theta \in \Sigma \Theta$. Otherwise let $x_{k_{1}}, x_{k_{2}}, \cdots, x_{k_{t}}\left(1 \leqq k_{1}<k_{2}<\cdots<k_{t} \leqq n\right)$ be the nodes of $\theta$ which are intersection nodes. We then put $\left(x_{1}, \cdots, x_{k_{1}}\right)$, $\left(x_{k_{1}}, \cdots, x_{k_{2}}\right), \cdots,\left(x_{k_{t}}, \cdots, x_{n}\right)$ in $\Sigma \Theta$. Notice that it may happen that $x_{k_{1}}=x_{1}$ so that $\left(x_{1}, \cdots, x_{k_{1}}\right)$ is the degenerate path $\left(x_{1}\right)$; likewise it may happen that $\left(x_{k_{t}}, \cdots, x_{n}\right)$ is the degenerate path $\left(x_{n}\right)$. The set $\Sigma \Phi$ is constructed in an analogous way.

Let In $\Theta \cup \operatorname{In} \Phi \subseteq X$ and Ter $\Theta \cup$ Ter $\Phi \subseteq Y$. There is then associated a bipartite graph, denoted by $G(\Theta, \Phi)$ in the following way. Let $X_{I}$, $Y_{T}, X^{\prime}, X^{\prime \prime}$ be pairwise node disjoint sets such that there are bijections $x \rightarrow x_{I}$ of $X$ to $X_{I}, y \rightarrow y_{T}$ of $Y$ to $Y_{T}$, and $x \rightarrow x^{\prime}$ and $x \rightarrow x^{\prime \prime}$ of Nod $\Theta \cap \operatorname{Nod} \Phi$ to $X^{\prime}$ and $X^{\prime \prime}$, respectively. The sets $X_{I} \cup X^{\prime}$ and $Y_{T} \cup X^{\prime \prime}$ (which are disjoint by our assumptions) comprise the nodes of $G(\Theta, \Phi)$. We now construct the edges. Corresponding to each path in $\Sigma(\Theta)$ there is to be an edge of $G(\Theta, \Phi)$. Let $\theta=\left(x=x_{1}, x_{2}, \cdots, x_{n}\right.$ $=y) \in \Theta$. If $\theta \in \Sigma(\Theta)$ we associate the edge $\left(x_{I}, y_{T}\right)$. Otherwise if $\left(x, \cdots, x_{k_{1}}\right),\left(x_{k_{1}}, \cdots, x_{k_{2}}\right), \cdots,\left(x_{k_{t}}, \cdots, y\right)$ are the subpaths of $\theta$ in $\Sigma(\Theta)$ we associate the edges $\left(x_{I}, x_{k_{1}}^{\prime \prime}\right),\left(x_{k_{1}}^{\prime}, x_{k_{2}}^{\prime \prime}\right), \cdots,\left(x_{k_{t}}^{\prime}, y_{T}\right)$. The collection of edges of $G(\Theta, \Phi)$ constructed in this way is denoted by $\tilde{\Sigma}(\Theta)$. In an analogous way the paths in $\Sigma(\Phi)$ give rise to a set $\tilde{\Sigma}(\Phi)$ of edges of $G(\Theta, \Phi)$. These are to be the only edges of $G(\Theta, \Phi)$. Observe that $\tilde{\Sigma}(\Theta)$, resp. $\widetilde{\Sigma}(\Phi)$, is a set of pairwise node disjoint edges of $G(\Theta, \Phi)$.

The rule of [3] can now be formulated:

If $\lambda$ is a (finite) collection of pairwise node disjoint edges of $G(\Theta, \Phi)$ with

$$
X^{\prime} \subseteq \operatorname{In} \lambda \subseteq X_{I} \cup X^{\prime}, \quad X^{\prime \prime} \subseteq \operatorname{Ter} \lambda \subseteq Y_{T} \cup X^{\prime \prime},
$$

then there is a (finite) collection $\Pi$ of pairwise node disjoint finite linear paths in $\Gamma$ with

$$
\text { In } \Pi=\left\{x: x_{I} \in \operatorname{In} \lambda\right\}, \quad \text { Ter } \Pi=\left\{y: y_{T} \in \operatorname{Ter} \lambda_{\}} .\right.
$$

As formulated in [3] the presence of cyclic paths (paths whose terminal node coincides with its initial node) is possible. The set $\Pi$ above is what remains after the cyclic paths have been discarded.

3. Let $X$ be an arbitrary set. A matroid [10], [11] on $X$ is a nonempty collection $M$ of subsets of $X$ such that (1) $A \in M, A^{\prime} \subseteq A$ imply $A^{\prime} \in M$ and (2) $A_{1}, A_{2} \in M,\left|A_{1}\right|+1=\left|A_{2}\right|<\infty$ imply there is an $x \in A_{2} \backslash A_{1}$ with $A_{1} \cup\{x\} \in M$. Those subsets of $X$ which are members of $M$ are called independent sets. A base of $M$ is a maximal in- 
dependent subset of $X$. If $|X|<\infty, M$ is a finite matroid; if one base of $M$ is finite, then all bases are finite and have the same cardinal number. Matroids with a finite base are called rank-finite matroids. If $S \subseteq X$, then $M_{S}=\{A \subseteq S: A \in M\}$ is a matroid on $S$, called the restriction of $M$ to $S$. If $M_{1}$ and $M_{2}$ are matroids on disjoint sets $X_{1}$ and $X_{2}$, respectively, then $M_{1} \oplus M_{2}$ is a matroid on $X_{1} \cup X_{2}$, called the direct sum of $M_{1}$ and $M_{2}$. The matroid on a set $X$ consisting of all subsets of $X$ is called the free matroid on $X$ and is denoted by $\rho(X)$. For more information on these matters, one may consult [10], [11], or [5].

There are a number of ways to induce matroids from given matroids. Let $G$ be a bipartite graph with node set $X \cup Y(X \cap Y=\varnothing)$ where each edge has initial node in $X$ and terminal node in $Y$. Let $M^{1}$ be a matroid on $Y$ and define a collection $M^{2}$ of subsets of $X$ by: $A \in M^{2}$ if and only if there is a set $\Pi$ of pairwise node disjoint edges with $\ln \Pi=A$ and Ter $\Pi \in M^{1}$. Then $M^{2}$ is a matroid on $X$ [7]. Those matroids obtained in this way when $\boldsymbol{M}^{1}$ is the free matroid on $Y$ are called transversal matroids [4]. We use the rule formulated in $\$ 2$ to give relatively simple proofs of the following theorems. But first we state and prove two lemmas.

If $M$ is a matroid on a set $X$ and $A \in M$, then the span of $A$ is the set $A \cup\{z \in X \backslash A: A \cup\{z\} \notin M\}$. If $Z$ is the span of $A$, then $A$ is a base of $M_{Z}$.

Lemma 1. Let $G$ be a bipartite graph with node set $X \cup Y(X \cap Y=\varnothing)$, and let $\boldsymbol{M}^{1}$ be a matroid on $Y$. Let $\Theta_{i}$ be a finite set of pairwise node disjoint edges of $G$ with In $\Theta_{i}=B_{i}$ and $\operatorname{Ter} \Theta_{i}=A_{i} \in M^{1}(i=1,2)$. Then one of the following two possibilities must occur.

(1) There exists an $x \in B_{2} \backslash B_{1}$ and a set $\Pi$ of pairwise node disjoint edges with In $\Pi=B_{1} \cup\{x\}$ and $A_{1} \cap A_{2} \subseteq$ Ter $\Pi \in M^{1}$.

(2) There exists a set $\Pi$ of pairwise node disjoint edges with In $\Pi=B_{1}$, $A_{1} \cap A_{2} \subseteq$ Ter $\Pi \in M^{1}$, and $A_{2} \subseteq Z$ where $Z$ is the span of Ter $\Pi$ (relative to the matroid $M^{1}$ ).

If $A_{2}$ is contained in the span of $A_{1}$ relative to $\boldsymbol{M}^{1}$, then we may take $\Pi=\Theta_{1}$ to satisfy possibility (2). Thus assume there is a $y \in A_{2} \backslash A_{1}$ with $A_{1} \cup\{y\} \in M^{1}$. The node $y$ determines a sequence of distinct edges which alternate in $\Theta_{2}$ and $\Theta_{1}$ and which either (i) ends with an edge of $\Theta_{2}$ with initial node $x \in B_{2} \backslash B_{1}$ or (ii) ends with an edge of $\Theta_{1}$ with terminal node $z \in A_{1} \backslash A_{2}$. Let $\Delta_{i}$ be the set of edges of $\Theta_{i}$ in this sequence $(i=1,2)$ and let $\Theta_{3}=\left\{\Theta_{1} \backslash \Delta_{1}\right\} \cup \Delta_{2}$. In case (i), $\Theta_{3}$ is a set of pairwise node disjoint edges of $G$ with In $\Theta_{3}=B_{1} \cup\{x\}$ and $A_{1} \cap A_{2}$ $\subseteq$ Ter $\Theta_{3}=A_{1} \cup\{y\} \in M^{1}$. We may then take $\Pi=\Theta_{3}$ to satisfy possibility (1). In case (ii), $\Theta_{3}$ is a set of pairwise node disjoint edges with 
In $\Theta_{3}=B_{1}, A_{1} \cap A_{2} \subseteq \operatorname{Ter} \Theta_{3}=\left\{A_{1} \backslash\{y\}\right\} \cup\{z\}=A_{3} \in M^{1}$ where $\mid A_{3}$ $\cap A_{2}|>| A_{1} \cap A_{2} \mid$. We may then replace $\Theta_{1}$ by $\Theta_{3}$ and repeat the above procedure. After a finite number of applications either we achieve case (i) and thus satisfy possibility (1) or we obtain a set $\Theta_{k}(k \geqq 3)$ of pairwise node disjoint edges with In $\Theta_{k}=B_{1}, A_{1} \cap A_{2}$ $\subseteq$ Ter $\Theta_{k}=A_{k} \in M^{1}$ where $A_{k} \subseteq A_{2}$. If $A_{2}$ is contained in the span of $A_{k}$ relative to $\boldsymbol{M}^{1}$, we may take $\Pi=\Theta_{k}$ to satisfy possibility (2). Otherwise one more application of the above procedure must lead to the occurrence of case (i) and thus to the satisfying of possibility (1).

Lemma 2. Let $\Gamma$ be a directed graph, and let $X, Y$ be subsets of the nodes of $\Gamma$. Assume that $M^{1}$ is a matroid on $Y$. Let $\Theta_{i}$ be a finite set of pairwise node disjoint, finite linear paths in $\Gamma$ with In $\Theta_{i}=B_{i} \subseteq X$ and Ter $\Theta_{i}=A_{i} \in M^{1}(i=1,2)$. Then one of the following two possibilities must occur.

(1) There exists an $x \in B_{2} \backslash B_{1}$ and a set $\Pi$ of pairwise node disjoint, finite linear paths in $\Gamma$ with In $\Pi=B_{1} \cup\{x\}$ and Ter $\Pi \in M^{1}$.

(2) There exists a set $\Pi$ of pairwise node disjoint, finite linear paths in $\Gamma$ with In $\Pi=B_{1}$, Ter $\Pi \in M^{1}$, and $A_{2} \subseteq Z$ where $Z$ is the span of Ter $\Pi$ (relative to the matroid $M^{1}$ ).

We deduce Lemma 2 from Lemma 1. Thus let $G\left(\Theta_{1}, \Theta_{2}\right)$ be the bipartite graph associated with $\Theta_{1}$ and $\Theta_{2}$ as in $\$ 2$. Because of the bijection from $Y$ to $Y_{T}$ we may consider $M^{1}$ as a matroid on $Y_{T}$. We then consider the matroid $M^{1} \oplus \odot\left(X^{\prime \prime}\right)$ on $Y_{T} \cup X^{\prime \prime}$. Then $\widetilde{\Sigma}\left(\Theta_{i}\right)$ is a collection of pairwise node disjoint edges in $G\left(\Theta_{1}, \Theta_{2}\right)$ with In $\tilde{\Sigma}\left(\Theta_{i}\right)$ $=\left(B_{i}\right)_{I} \cup X^{\prime}$ and Ter $\tilde{\Sigma}\left(\Theta_{i}\right)=\left(A_{i}\right)_{T} \cup X^{\prime \prime} \in M^{1} \oplus \rho\left(X^{\prime \prime}\right)(i=1,2)$. We now apply Lemma 1 to conclude one of the following two possibilities must occur.

(3) There exists $x_{I} \in\left(B_{2}\right)_{I} \backslash\left(B_{1}\right)_{I}$ and a set $\lambda$ of pairwise node disjoint edges of $G\left(\Theta_{1}, \Theta_{2}\right)$ with $\ln \lambda=\left(B_{1}\right)_{I} \cup\left\{x_{I}\right\} \cup X^{\prime}$ and $X^{\prime \prime} \subseteq$ Ter $\lambda$ $\in M^{1} \oplus \odot\left(X^{\prime \prime}\right)$.

(4) There exists a set $\lambda$ of pairwise node disjoint edges of $G\left(\Theta_{1}, \Theta_{2}\right)$ with In $\lambda=\left(B_{1}\right)_{I} \cup X^{\prime}, X^{\prime \prime} \subseteq$ Ter $\lambda \in M^{1} \oplus \mathcal{P}\left(X^{\prime \prime}\right)$, and $\left(A_{2}\right)_{T} \cup X^{\prime \prime} \subseteq Z$ where $Z$ is the span of Ter $\Pi$ (relative to the matroid $M^{1} \oplus \odot\left(X^{\prime \prime}\right)$ ).

By the rule of $\S 2$, (3) leads to an $x \in B_{2} \backslash B_{1}$ and a set $\Pi$ of pairwise node disjoint, finite linear paths satisfying (1), while (4) leads to a set II satisfying (2).

We use Lemma 2 now to prove Theorems 1 and 2.

Theorem 1. Let $\Gamma$ be a directed graph, and let $X, Y$ be subsets of the nodes of $\Gamma$. Let $\boldsymbol{M}^{1}$ be a matroid on $Y$ and define a collection $\boldsymbol{M}^{2}$ of subsets of $X$ by: $B \in M^{2}$ if and only if there is a set $\Theta$ of pairwise node disjoint, 
finite linear paths with In $\Theta=B$, Ter $\Theta \in M^{1}$. Then $\boldsymbol{M}^{2}$ is a matroid on $X$.

Property (1) for matroids being clear, we need to verify property (2). Thus let $B_{1}, B_{2} \in M^{2}$ with $\left|B_{1}\right|=k,\left|B_{2}\right|=k+1$, and let $\Theta_{i}$ be a set of pairwise node disjoint, finite linear paths in $\Gamma$ with In $\Theta_{i}=B_{i}$, Ter $\Theta_{i}=A_{i} \in M^{1}(i=1,2)$. We now apply Lemma 2 and observe that possibility (2) can now not occur. For if it did we would have $A_{2}$ contained in the span of Ter $\Pi$ (relative to the matroid $\boldsymbol{M}^{1}$ ) where $A_{2} \in M$ and $\left|A_{2}\right|=k+1$ but $\mid$ Ter $\Pi \mid=k$. This contradicts the defining properties of matroids. Hence possibility (1) of Lemma 2 must occur, and we conclude that there is an $x \in B_{2} \backslash B_{1}$ with $B_{1} \cup\{x\} \in M^{2}$. Hence $\boldsymbol{M}^{2}$ is a matroid on $X$.

With reference to Theorem 1 , the matroid $M^{2}$ (on $X$ ) is said to be induced from the matroid $M^{1}$ (on $Y$ ) by the directed graph $\Gamma$. If $M^{1}$ is the free matroid on $Y$, this result has been proved in [8] and [9] (where a matroid is referred to as a pre-independence structure).

TheOREм 2. Let $\Gamma$ be a directed graph, and let $X, Y$ be subsets of the nodes of $\Gamma$. Suppose the matroid $M^{2}$ on $X$ is induced from the rank-finite matroid $M^{1}$ on $Y$ by $\Gamma$. If $B$ is any base of $M^{2}$ and $\Theta$ is any set of pairwise node disjoint, finite linear paths in $\Gamma$ with $\ln \Theta=B$, Ter $\Theta=A$ $\in M^{1}$, then $\boldsymbol{M}^{2}$ (on $X$ ) is induced from $\boldsymbol{M}_{Z}^{1}$ (on $Z$ ) by $\Gamma$ where $Z$ is the span of $A$ relative to the matroid $\boldsymbol{M}^{1}$.

The corresponding result for bipartite graphs is derived in [2] when $\boldsymbol{M}^{1}$ is the free matroid on $Y(Z$ is then $A)$ and is generalized in [6]. If $M^{3}$ is the matroid induced on $X$ from $M_{Z}^{1}$ on $Z$ by $\Gamma$, then it is clear that $\boldsymbol{M}^{3} \subseteq \boldsymbol{M}^{2}$. Thus we need to show that $\boldsymbol{M}^{2} \subseteq \boldsymbol{M}^{3}$. This is a consequence of the following argument.

Let $B_{i}$ be a base of $M^{2}$ and let $\Theta_{i}$ be a set of pairwise node disjoint, finite linear paths in $\Gamma$ with $\operatorname{In} \Theta_{i}=B_{i}$, Ter $\Theta_{i}=A_{i} \in M^{1}(i=1,2)$. We now apply Lemma 2 and observe that possibility (1) can now not occur. For, if it did, it would have as a consequence that there is an $x \in B_{2} \backslash B_{1}$ with $B_{1} \cup\{x\} \in \boldsymbol{M}^{2}$. This, however, contradicts the assumption that $B_{1}$ is a base of $\boldsymbol{M}^{2}$. Hence possibility (2) of Lemma 2 must occur. Thus there is a set $\Pi$ of pairwise node disjoint, finite linear paths in $\Gamma$ with In $\Pi=B_{1}$, Ter $\Pi \in M^{1}$, and $A_{2} \subseteq Z$ where $Z$ is the span of Ter $\Pi$ (relative to $\boldsymbol{M}^{1}$ ). But $A_{2}$, Ter $\Pi \in \boldsymbol{M}^{1}$ with $\left|A_{2}\right|=\left|B_{2}\right|$ $=\left|B_{1}\right|=\mid$ Ter $\Pi \mid$. But this means that Ter $\Pi$ is contained in the span of $A_{2}$ (relative to $\boldsymbol{M}^{1}$ ). Thus we have a set $\Pi$ of pairwise node disjoint, finite linear paths in $\Gamma$ with In $\Pi=B_{1}$ and Ter $\Pi$ contained in the span 
of $A_{2}$ (relative to $\boldsymbol{M}^{2}$ ). Thus $\boldsymbol{M}^{2}$ (on $X$ ) is induced from $\boldsymbol{M}_{Z_{2}}^{1}$ (on $Z_{2}$ ) where $Z_{2}$ is the span of $A_{2}$ (relative to $\boldsymbol{M}^{2}$ ).

Let $\boldsymbol{M}$ be a matroid on a set $X$. Then $\boldsymbol{M}$ has been called base orderable if given any two bases $B_{1}, B_{2}$ there is a bijection $\sigma: B_{1} \rightarrow B_{2}$ with $\left\{B_{1} \backslash\{x\}\right\} \cup\{\sigma(x)\}$ and $\left\{B_{2} \backslash\{\sigma(x)\}\right\} \cup\{x\}$ bases for all $x \in B_{1}$. Of course, the bijection $\sigma$ must be the identity on $B_{1} \cap B_{2}$. If in addition the bijection $\sigma$ can be chosen so that $\left\{B_{1} \backslash A\right\} \cup \sigma(A)$ and $\left\{B_{2} \backslash \sigma(A)\right\}$ $\cup A$ are bases for all $A \subseteq B_{1}$, then $\boldsymbol{M}$ will be called strongly base orderable. (This definition may be unnecessary, for to our knowledge all known base orderable matroids are strongly base orderable. In what follows we require the seemingly stronger property.) It was proved in [2] that all transversal matroids are base orderable and indeed strongly base orderable. In [1] it was shown that not all matroids are base orderable, so that this property gives a true necessary condition for a matroid to be a transversal matroid. The result that transversal matroids are strongly base orderable was generalized in [6] to include all matroids induced from strongly base orderable matroids by a bipartite graph. Obviously a free matroid is strongly base orderable, and it is readily verified that a restriction of a strongly base orderable matroid is strongly base orderable. To show that a matroid induced from a strongly base orderable matroid by a directed graph is also strongly base orderable, we require the following lemma.

Lемма 3. Let $G$ be a bipartite graph with node set $X \cup Y(X \cap Y=\varnothing)$, and let the matroid $M^{2}$ on $X$ be induced by $G$ from the strongly base orderable, rank-finite matroid $M^{1}$ on $Y$. If $\Theta_{i}$ is a set of pairwise node disjoint edges of $G$ with In $\Theta_{i}=B_{i}$, a base of $\boldsymbol{M}^{2}$, and Ter $\Theta_{i}=A_{i} \in M^{1}(i=1,2)$, then there is a bijection $\rho: B_{1} \backslash B_{2} \rightarrow B_{2} \backslash B_{1}$ such that for all $A \subseteq B_{1} \backslash B_{2}$ there exists a set $\Theta_{i}(A)$ of pairwise node disjoint edges of $G(i=1,2)$ with In $\Theta_{1}(A)=\left\{B_{1} \backslash A\right\} \cup_{\rho}(A), \quad A_{1} \cap A_{2} \subseteq$ Ter $\Theta_{1}(A) \in M^{1} \quad$ and In $\Theta_{2}(A)=\left\{B_{2} \backslash \rho(A)\right\} \cup A, A_{1} \cap A_{2} \subseteq \operatorname{Ter} \Theta_{2}(A) \in M^{1}$.

Using Theorem 2 and the fact that the restriction of a strongly base orderable matroid is strongly base orderable, we conclude that we may assume that $A_{i}$ is a base of $M^{1}(i=1,2)$. Let $\sigma: A_{1} \backslash A_{2} \rightarrow A_{2} \backslash A_{1}$ be a bijection with $\left\{A_{1} \backslash W\right\} \cup \sigma(W)$ and $\left\{A_{2} \backslash \sigma(W)\right\} \cup W$ bases of $\boldsymbol{M}^{1}$ for all $W \subseteq A_{1} \backslash A_{2}$. We define a bijection $\rho: B_{1} \backslash B_{2} \rightarrow B_{2} \backslash B_{1}$ as follows. Let $x \in B_{1} \backslash B_{2}$. Then $x$ determines a sequence of edges which alternate in $\Theta_{1}$ and $\Theta_{2}$ and which ends either with an edge of $\Theta_{2}$ with initial vertex $y \in B_{2} \backslash B_{1}$ or with an edge of $\Theta_{1}$ with terminal vertex $w_{1} \in A_{1} \backslash A_{2}$. In the former case we define $\rho(x)=y$. In the latter case we consider $\sigma\left(w_{1}\right) \in B_{2} \backslash A_{2}$ which determines a sequence of edges alternating in $\Theta_{2}$ and $\Theta_{1}$ that ends either with an edge of $\Theta_{2}$ with initial node $y \in B_{2} \backslash A_{2}$ 
or with an edge of $\Theta_{1}$ with terminal node $w_{2} \in A_{1} \backslash A_{2}$ where $w_{1} \neq w_{2}$. In the first case we define $\rho(x)=y$; in the second case we repeat with $\sigma\left(w_{2}\right)$ in place of $\sigma\left(w_{1}\right)$. After a finite number of steps we arrive at a finite sequence $w_{1}, w_{2}, \cdots, w_{k}(k \geqq 1)$ of distinct nodes of $A_{1} \backslash A_{2}$ such that the sequence of edges determined by $\sigma\left(w_{k}\right)$ which alternates in $\Theta_{2}$ and $\Theta_{1}$ ends with an edge of $\Theta_{2}$ with initial node $y \in B_{2} \backslash B_{1}$. We then define $\rho(x)=y$. Let $\Delta_{i}(x)$ be the set of edges of $\Theta_{i}$ encountered in defining $\rho(x)(i=1,2)$ and let $W(x)=\left\{w_{1}, w_{2}, \cdots, w_{k}\right\}$. (Note that $W(x)$ may be empty.) Since $\rho$ is a bijection, it is readily verified that $\left(\Delta_{1}(x) \cup \Delta_{2}(x): x \in B_{1} \backslash B_{2}\right)$ and $\left(W(x): x \in B_{1} \backslash B_{2}\right)$ are both families of pairwise disjoint sets. In particular, $\rho: B_{1} \backslash B_{2} \rightarrow B_{2} \backslash B_{1}$ is an injection and thus a bijection. If $A \subseteq B_{1} \backslash B_{2}$, then

$$
\begin{aligned}
& \Theta_{1}(A)=\left\{\Theta_{1} \backslash \bigcup_{x \in A} \Delta_{1}(x)\right\} \cup\left\{\bigcup_{x \in A} \Delta_{2}(x)\right\}, \\
& \Theta_{2}(A)=\left\{\Theta_{2} \backslash \bigcup_{x \in A} \Delta_{2}(x)\right\} \cup\left\{\bigcup_{x \in A} \Delta_{1}(x)\right\}
\end{aligned}
$$

are each pairwise node disjoint sets of edges of $G$ with

$$
\begin{aligned}
\operatorname{In} \Theta_{1}(A) & =\left\{B_{1} \backslash A\right\} \cup \rho(A), \\
A_{1} \cap A_{2} \subseteq \operatorname{Ter} \Theta_{1}(A) & =\left\{A_{1} \backslash \bigcup_{x \in A} W(x)\right\} \cup \sigma\left(\bigcup_{x \in A} W(x)\right) \in M^{1} . \\
\operatorname{In} \Theta_{2}(A) & =\left\{B_{2} \backslash \rho(A)\right\} \cup A, \\
A_{1} \cap A_{2} \subseteq \operatorname{Ter} \Theta_{2}(A) & =\left\{A_{2} \backslash \sigma\left(\bigcup_{x \in A} W(x)\right)\right\} \cup\left\{\bigcup_{x \in A} W(x)\right\} \in M^{1} .
\end{aligned}
$$

This establishes the lemma.

THEOREM 3. Let $\boldsymbol{M}^{1}$ be a strongly base orderable, rank-finite matroid on a set $Y$. If the matroid $M^{2}$ on $X$ can be induced from $M^{1}$ by a directed graph $\Gamma$, then $\boldsymbol{M}^{2}$ is also strongly base orderable.

Let $\Theta_{i}$ be a set of pairwise node disjoint, finite linear paths in $\Gamma$ with In $\Theta_{i}=B_{i}$, a base of $\boldsymbol{M}^{2}$, and Ter $\Theta_{i}=A_{i} \in \boldsymbol{M}^{1}(i=1,2)$. Let $G\left(\Theta_{1}, \Theta_{2}\right)$ be the bipartite graph associated with $\Theta_{1}$ and $\Theta_{2}$ as in $\S 2$, and regard $\boldsymbol{M}^{1}$ as a matroid on $Y_{T}$. Consider then the matroid $\boldsymbol{M}^{1}$ $\oplus \odot\left(X^{\prime \prime}\right)$ on $Y_{T} \cup X^{\prime \prime}$. Then $\tilde{\Sigma}\left(\Theta_{i}\right)$ is a set of pairwise node disjoint edges of $G\left(\Theta_{1}, \Theta_{2}\right)$ with In $\tilde{\Sigma}\left(\Theta_{i}\right)=\left(B_{i}\right)_{I} \cup X^{\prime}$, Ter $\tilde{\Sigma}\left(\Theta_{i}\right)=\left(A_{i}\right)_{T} \cup X^{\prime \prime}$ $(i=1,2)$. Since from Theorem 2 we may assume that $A_{1}$ and $A_{2}$ are bases of $\boldsymbol{M}^{1}$, it follows by a simple cardinality argument that $\left(B_{i}\right)_{I}$ $\cup X^{\prime}$ is a base of the matroid induced on $X_{I} \cup X^{\prime}$ from $M^{1} \oplus \mathcal{P}\left(X^{\prime \prime}\right)$ by 
$G\left(\Theta_{1}, \Theta_{2}\right)$. Using Lemma 3 , we conclude that there is a bijection $\rho_{I}:\left(B_{1}\right)_{I} \backslash\left(B_{2}\right)_{I} \rightarrow\left(B_{2}\right)_{I} \backslash\left(B_{1}\right)_{I}$ such that for all $A_{I} \subseteq\left(B_{1}\right)_{I} \backslash\left(B_{2}\right)_{I}$ there are sets $\lambda_{1}(A), \lambda_{2}(A)$ of pairwise node disjoint edges of $G\left(\Theta_{1}, \Theta_{2}\right)$ with

$$
\begin{aligned}
X^{\prime} & \subseteq \operatorname{In} \lambda_{1}(A)=\left\{\left(B_{1}\right)_{I} \backslash A_{I}\right\} \cup \rho_{I}\left(A_{I}\right), \\
X^{\prime \prime} & \subseteq \operatorname{Ter} \lambda_{1}(A) \in M^{1} \oplus \odot\left(X^{\prime \prime}\right), \\
X^{\prime} & \subseteq \operatorname{In} \lambda_{2}(A)=\left\{\left(B_{2}\right)_{I} \backslash \rho_{I}\left(A_{I}\right)\right\} \cup A_{I}, \\
X^{\prime \prime} & \subseteq \operatorname{Ter} \lambda_{2}(A) \in M^{1} \oplus \odot\left(X^{\prime \prime}\right) .
\end{aligned}
$$

Thus by the rule of $\S 2$ there is a bijection $\rho: B_{1} \backslash B_{2} \rightarrow B_{2} \backslash B_{1}$ with the property that for all $A \subseteq B_{1} \backslash B_{2}$ there exist sets $\Pi_{1}(A), \Pi_{2}(A)$ of pairwise node disjoint finite linear paths in $\Gamma$ with

$$
\begin{aligned}
& \text { In } \Pi_{1}(A)=\left\{B_{1} \backslash A\right\} \cup \rho(A), \quad \text { Ter } \Pi_{1} \in \boldsymbol{M}^{1}, \\
& \text { In } \Pi_{2}(A)=\left\{B_{2} \backslash \rho(A)\right\} \cup A, \quad \text { Ter } \Pi_{2} \in M^{1} \text {. }
\end{aligned}
$$

Hence $\boldsymbol{M}^{2}$ is a strongly base orderable matroid on $X$.

CoRollaRY. If a matroid $M$ on $X$ can be induced from a free matroid by a directed graph, then $M$ is strongly base orderable.

This is a special case of the theorem, since a free matroid is trivially strongly base orderable.

On six or fewer elements there are up to isomorphism only two matroids which are not transversal matroids. These are the cycle matroids [10] of the complete graph $K_{4}$ on four nodes, $\boldsymbol{M}\left(K_{4}\right)$, and the cycle matroid of the graph obtained from a triangle by repeating each edge. It is easy to verify that the latter matroid is strongly base orderable and indeed can be induced from a free matroid by a directed graph. The matroid $M\left(K_{4}\right)$ is, however, not base orderable, as is easily verified, and hence cannot be induced from a free matroid by any directed graph. We may conclude that on six or fewer elements there is up to isomorphism exactly one matroid, $\boldsymbol{M}\left(K_{4}\right)$, which cannot be induced from a free matroid by a directed graph; similarly, there is exactly one matroid on six or fewer elements, namely $\boldsymbol{M}\left(K_{4}\right)$, which is not base orderable (or strongly base orderable).

\section{REFERENCES}

1. R. A. Brualdi, Comments on bases in dependence structures, Bull. Austral. Math. Soc. 1 (1969), 161-167. MR 40 \#146.

2. R. A. Brualdi and E. B. Scrimger, Exchange systems, matchings, and transversals, J. Combinatorial Theory 5 (1968), 244-257. MR 38 \#55.

3. R. A. Brualdi and J. S. Pym, A general linking theorem in directed graphs, 
edited by L. Mirsky, Studies in Pure Mathematics, Academic Press, London and New York, 1971.

4. J. Edmonds and D. R. Fulkerson, Transversals and matroid partition, J. Res. Nat. Bur. Standards Sect. B 69B (1965), 147-153. MR 32 \#5531.

5. F. Harary and D. J. A. Welsh, Matroids versus graphs: The many facets of graph theory, Lecture Notes in Math., no. 110, Springer-Verlag, Berlin, pp. 155-170.

6. J. H. Mason, Representations of independence spaces, $\mathrm{Ph} . \mathrm{D}$. Dissertation, University of Wisconsin, Madison, Wis., 1969.

7. $\mathrm{H}$. Perfect, Independence spaces and combinatorial problems, Proc. London Math. Soc. (3) 19 (1969), 17-30. MR 39 \#2649.

8. —_, Applications of Menger's graph theorem, J. Math. Anal. Appl. 22 (1968), 96-111. MR 37 \#93.

9. J. S. Pym and H. Perfect, Submodular functions and independence structures, J. Math. Anal. Appl. 30 (1970), 1-37.

10. W. T. Tutte, Lectures on matroids, J. Res. Nat. Bur. Standards Sect. B 69B (1965), 1-47. MR 31 \#4023.

11. H. Whitney, On the abstract properties of linear dependence, Amer. J. Math. 57 (1935), 509-533.

University of Wisconsin, Madison, Wisconsin 53706 